Service social

\title{
La parole en public : savoir être et savoir faire, par Jacques Vermette et Richard Cloutier, Sainte-Foy, Les presses de l’Université Laval, 1992, 208 pages.
}

\section{René Auclair}

Volume 42, numéro 1, 1993

Culture et intervention

URI : https://id.erudit.org/iderudit/706613ar

DOI : https://doi.org/10.7202/706613ar

Aller au sommaire du numéro

Éditeur(s)

École de service social de l'Université Laval

ISSN

1708-1734 (numérique)

Découvrir la revue

Citer ce compte rendu

Auclair, R. (1993). Compte rendu de [La parole en public : savoir être et savoir faire, par Jacques Vermette et Richard Cloutier, Sainte-Foy, Les presses de l’Université Laval, 1992, 208 pages.] Service social, 42(1), 172-173.

https://doi.org/10.7202/706613ar d'utilisation que vous pouvez consulter en ligne.

https://apropos.erudit.org/fr/usagers/politique-dutilisation/ 


\section{LA PAROLE EN PUBLIC: SAVOIR ÊTRE ET SAVOIR FAIRE}

Jacques Vermette et Richard Cloutier,

Sainte-Foy, Les Presses de l'Université Laval, 1992, 208 pages.

Comme l'annonce le feuillet publicitaire, «neuf spécialistes (oto-rhino-laryngologiste-phoniatre, pharmacologue, linguiste, psychologues et communicateurs) fournissent les informations nécessaires concernant les enjeux physiologiques de la parole en public jusqu'à ses implications psychologiques, en passant par la palette des éléments à mettre en place pour parler en public efficacement. Des moyens concrets sont suggérés pour atténuer les effets de la timidité et du trac, ou encore de certains aliments et médicaments, pour projeter les sons avec qualité, pour tirer profit du charisme séducteur et pour s'exprimer avec spontanéité ».

Dans le premier chapitre portant sur l'appareil vocal, François Parent, oto-rhino-laryngologiste-phoniatre, nous présente et nous explique I'appareil vocal sous I'angle anatomique et physiologique. À cela, il ajoute les anomalies de l'appareil vocal et nous fait faire des liens entre la santé et I'hygiène vocale.

François Doré, professeur en pharmacologie et toxicologie, nous informe dans le deuxième chapitre des effets potentiellement nuisibles ou bénéfiques de certains médicaments ou nutriments sur la performance orale, ce qui implique un retour intéressant sur le système nerveux en relation avec ces substances.

Claude Rochette, professeur en phonétique, nous suggère, dans le troisième chapitre, d'utiliser une grille d'analyse pour évaluer notre propre expression orale:

1. I'appréciation du débit;

2. la valeur de l'intonation;

3. les variations du rythme;

4. I'exploitation de l'intensité de la voix;

5. I'enchaînement des sons;

6. I'articulation des consonnes;

7. la production des voyelles;

8. les phénomènes d'hypercorrection;

9. la prononciation des mots empruntés.

Richard Cloutier, professeur en psychologie, aborde le trac et la timidité, dans le quatrième chapitre, comme deux variantes de l'anxiété sociale, c'est-à-dire l'anxiété causée par la peur de ne pas avoir les moyens d'atteindre une certaine qualité dans la performance oratoire, la crainte de ne pas réussir à donner l'image désirée devant les autres. La présentation par l'auteur des stratégies de contrôle de l'image sociale est une vraie mine d'or. 
Dans le cinquième chapitre, Denis Pelletier, professeur en orientation et counseling, nous fait voir d'une façon très personnelle que la parole en public vise toujours en définitive à faire connaître son point de vue, sa manière personnelle de voir les choses même dans une communication scientifique.

Claude Cossette, professeur en communication, aborde dans le chapitre suivant une question fondamentale, celle du charisme séducteur. Le charisme est-il un talent qui se cultive ou un don? L'auteur croit pour sa part que l'on naît avec un talent charismatique, mais que, comme tous les talents, le don charismatique se cultive. C'est ce qu'il tente de nous démontrer dans son exposé.

Dans le septième chapitre, Monique Brillon et Jocelyne Taillon, psychologues, nous révèlent les aspects cachés de la parole, tant corporels et affectifs qu'émotionnels. Ces aspects comportent des dimensions à la fois conscientes et inconscientes, en constante interaction, à notre insu, et influencent grandement notre performance oratoire. Les divers scénarios possibles de la prise de parole en public, présentés par les auteures, sont des repères aussi importants que les phares dans la nuit.

Jacques Vermette, professeur en communication et information, avec ses variations sur parole, dans le dernier chapitre, nous suggère que la parole en public est une musique dont nous sommes le chef d'orchestre. La transmission par l'oral est d'abord une affaire de relations humaines dont le message, surtout s'il est lu, est le prétexte. La grille d'évaluation présentée en conclusion par l'auteur permet de nous situer tant par rapport aux paramètres que sont les attitudes, les pensées, le non-verbal, les sons, les mots et le matériel que par rapport à leurs éléments constitutifs, tout comme une partition d'orchestre. Quant aux exercices pour les voyelles et les consonnes proposés à la toute fin, ils font partie du métier.

Voilà un livre qui bouscule certains préjugés, mais qui surtout a le mérite d'organiser dans un modèle relativement simple un grand nombre de faits disparates et dispersés. Le non-spécialiste y trouvera donc son profit et découvrira un ensemble intégré et accessible de connaissances de base, alors que les plus avertis auront plaisir à pousser plus loin le questionnement et les débats de fond qu'il soulève.

René AUCLAIR

École de service social

Université Laval 